\title{
Optimal Design for an Extruder Head Runner Based on Response Surface Method and Simulated Annealing Algorithm
}

\author{
Haichao Zhou $\mathbb{D}^{1,2}$ Zhen Jiang, ${ }^{1}$ Wenchao Li $\mathbb{D}^{1},{ }^{1}$ Guolin Wang, ${ }^{1}$ and Yongjie $\mathrm{Tu}^{2}$ \\ ${ }^{1}$ School of Automotive and Traffic Engineering, Jiangsu University, Zhenjiang 212013, China \\ ${ }^{2}$ Tire Institute, Double Coin Holdings Ltd, Shanghai 201109, China \\ Correspondence should be addressed to Wenchao Li; 1000002146@ujs.edu.cn
}

Received 2 March 2018; Accepted 26 April 2018; Published 27 May 2018

Academic Editor: Domenico Acierno

Copyright (C) 2018 Haichao Zhou et al. This is an open access article distributed under the Creative Commons Attribution License, which permits unrestricted use, distribution, and reproduction in any medium, provided the original work is properly cited.

\begin{abstract}
The head runner of a rubber extruder is important for controlling rubber flow and improving extrudate quality. To clarify the effect of the structure parameters of the head runner of a doubleplex tread extruder on extrudate quality and obtain high-quality rubber extrusions, a finite element model of the down head runner was established. The extrusion process was analyzed through numerical simulations, wherein the Bird-Carreau constitutive equation and Navier slip law were used along with some computational methods, such as quadratic interpolation of velocity and linear interpolation of pressure and viscosity. The Newton iteration algorithm was used for numerical calculations. The mean-square deviation of velocity (SDV) of rubber flow in the outlet cross section was selected as the evaluation objective. A Placket-Burman design was used to select three key factors-angles $A$ and $B$ and outlet width $D$-from among eight runner structure parameters affecting the velocity variance. By using central composite design (CCD), the quadratic response surface model using the three key factors was established, and the influence law of a combination of the three key factors on SDV was obtained. The response surface model was optimized using the simulated annealing (SA) algorithm, and the optimal key factors of the head runner were obtained. The optimal runner design realizes a more uniform velocity distribution in the outlet cross section. Furthermore, a comparison of the simulated flow velocities of the original and optimal head runners at different inlet flow ratios and temperatures indicates that the optimal head runner flow velocity improves the extrusion quality. Thus, an optimal runner with optimal key factors was manufactured. Test results of the rubber flow state indicated that the flow is regular and that warping disappears. The proposed optimization strategy can be used practically for improving the head runner design, shortening the product development cycle, and reducing the production cost.
\end{abstract}

\section{Introduction}

Tires are a core component of automobiles that affect automotive performance. Thus, the "Made in China 2025" plan classified automobile industry as one of the top ten "strongly pushed development of key breakthroughs in the field." The China Rubber Industry Association has targeted developments in tire manufacturing technology under China's 13th Five-Year Plan.

The tread quality realized by the extrusion process considerably affects tire performance [1]. At present, tread products are mainly produced using rubber extruders. To ensure the uniformity of rubber extruder products, the head runner structure is particularly crucial. Traditional head runner designs are created mainly through the trial-and-error method, resulting in high costs and long product development cycles. Furthermore, experiments cannot clarify the rubber flow state inside the head runner, making it difficult to propose improvements to the head runner structure.

Rubber exhibits die swell, melt fracture, and deformation distortion in the extrusion process, caused by stretching, extrusion, shear stress, and temperature [2,3]. The final quality of extruded products depends on the process conditions and extrusion shape. Furthermore, extrudate quality can be considerably improved by optimizing extruder head runner structure parameters [4]. Sun et al. found that differences in the thickness ratio of each layer resulted in differences in the properties of extruded products made of composite materials [5]. Dooley and Hilton studied the influence of runner geometry on the extrusion quality to reduce the effect of 
viscosity on the thickness of the extruded layer and found that different runner structures caused velocity rearrangement at the interface, resulting in polymer coextrusion producing layers of uneven thickness [6]. Musil and Zatloukal explored the effects of gradual expansion, chamfering, and tapering on flow stability at the outlet of the head runner [7]. Elgeti et al. used a numerical method to identify the geometric factors affecting the product quality by controlling various parameters to obtain an optimum head runner structure [8]. Zhong et al. studied the UHMWPE plunger extruder runner to determine the impact of the geometrical parameters of the buffer section and transition section on the rubber flow; they found that decreasing the buffer cross section and transition section slope improved the product quality [9].

Tread rubber extrusion process can be simulated using the finite element method, and this process can be evaluated in detail from the flow characteristics to design a runner. Lin et al. analyzed the flow state of the compound in the runner by using Fluent software and analyzed the impact of the variation of the runner choke block on the flow behavior and export speed of the compound, as well as improved the lower part of the choke block and quality of extruded plastic material [10]. Dai et al. compared the uniformity of the fluid extrusion velocity distribution of the rubber-sealing die, determined the judge feasibility of the runner design, and optimized the runner [11]. Liang et al. proposed a method for increasing the height of the slit area and for expanding the die inlet to effectively resolve uneven velocity distribution in the initial mold design as well as to improve rubber flow in the runner and the extrusion quality [12]. For a doubleplex tread extruder runner, the rubber flow rate at the outlet horizontal width must be uniform. However, rubber flows in the runner are affected by the geometry of the choke block and the head runner. Thus far, studies on the extrusion of tire rubber have mainly focused on analyzing the traction speed, flow, and other process parameters; few studies have investigated the effect of runner geometry parameters on extrusion quality. We cannot say for sure the influence order of head runner structure parameters on rubber extruded quality and still have difficulty putting quick effect optimization method to improve runner structure and promote rubber extrudate quality.

The present study focuses on the down head runner structure of a doubleplex tread extruder. The isothermal Newtonian fluid model is used to simulate rubber flow in the runner. First, the mean-square deviation of velocity (SDV) of rubber flow in the outlet cross section of the runner is used as the evaluation index, and the key design parameters of the head runner and choke block structure are obtained using the Plackett-Burman (PB) design method. PB analysis results show that the angles $A$ and $B$ and outlet width $D$ are three key factors influencing the extrusion quality. Second, central composite design (CCD) is used to build the response surface model of the effect of key parameters on extrusion quality. Third, the response surface model is optimized through the simulated annealing (SA) algorithm, and the optimum head runner design is established. The rubber extrusion quality in the original runner and the optimal runner is compared at different flow rates and different temperatures, and the results show that the optimized runner improves the extrusion quality. The test results of the rubber flow state indicated that the flow is regular and that warping disappears.

\section{Computational Model and Method}

2.1. Geometric Model and Finite Element Model. This study focuses on the down head runner of a doubleplex tread extruder. Figure 1 shows the geometry of the head runner. When the rubber flows into the head runner from the extruder screw, the fluid is split into two sections and flows into the respective calibration sections by the choke block. Because of the rheological properties of the rubber polymer and contraction of the cross section of both sides of the runner, the rubber streamline is not parallel and the flow velocity is not uniform, resulting in a tapered shape converging at the outlet. The main aim then is to adjust the flow distribution and improve the flow pressure to ensure the uniformity of the flow rate at the outlet.

The runner has an inlet width of $500 \mathrm{~mm}$ and outlet width of $600 \mathrm{~mm}$, with a choke block width of $460 \mathrm{~mm}$; the runner outlet also has two $70 \mathrm{~mm}$-wide sides. Because the runner has plane symmetry, only one symmetrical half of the runner is considered in the finite element model, as shown in Figure 2(a). Figure 2(b) shows the boundary conditions. To ensure that the inlet pressure has uniform distribution, a $200 \mathrm{~mm}$ extending runner is used at the inlet. To develop the rubber flow completely, a $250 \mathrm{~mm}$ free expansion zone is used. Eight runner parameters, $A$ to $H$, are selected to analyze their effect on extruder uniformity. $A$ and $B$ are choke block angles, $C$ is the choke block transitional fillet radius, $D$ is the outlet width, $E$ and $F$ are the sidewall transitional fillet radius, $H$ is the choke block top fillet radius, and $G$ is the inlet width. Tetrahedral elements are used in the head runner and triangular prism elements in the free expansion zone and extending runner zone. The size of elements should be set differently by considering the time cost and accuracy of the simulation. The elements in the head runner area are smaller in size than are those in the free zone and extending runner, and rubber flow in this area is fully developed. Therefore, meshing in the head runner is refined, and the element size is $1 \mathrm{~mm}$. The free zone and extending runner are meshed coarsely with an element size of $3 \mathrm{~mm}$.

2.2. Control Equation and Constitutive Equation. The tread rubber extrusion process follows the conservation of mass and momentum. Assuming that tread rubber is an incompressible isothermal non-Newtonian fluid flow [13], that the flow is stable and laminar, and that the influence of gravity, inertia force, and temperature on the flow is ignored, the rubber flow control equation in Euler space coordinates can be given by the following:

$$
\begin{aligned}
\nabla \cdot v & =0, \\
-\nabla p+\nabla \cdot \tau & =0 .
\end{aligned}
$$

Here, $\nabla$ is the Hamiltonian operator, $v$ the velocity vector, $p$ the static pressure, and $\tau$ the deviatoric stress tensor. 


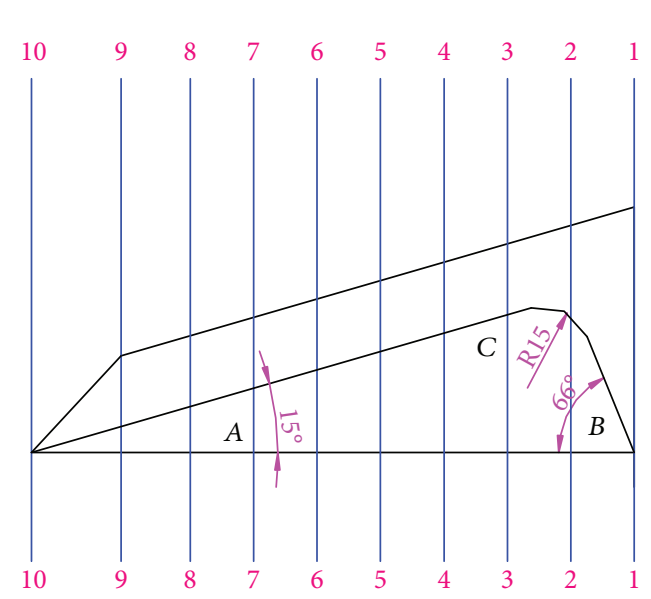

(a) Main view

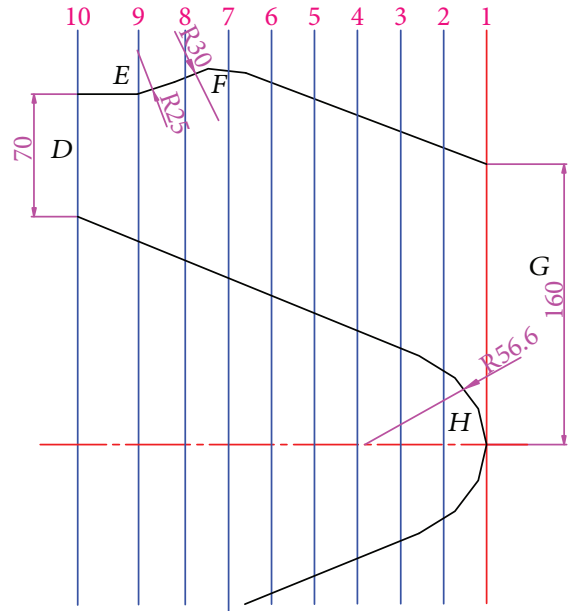

(b) Vertical view

FIgURE 1: Structure of the head runner.

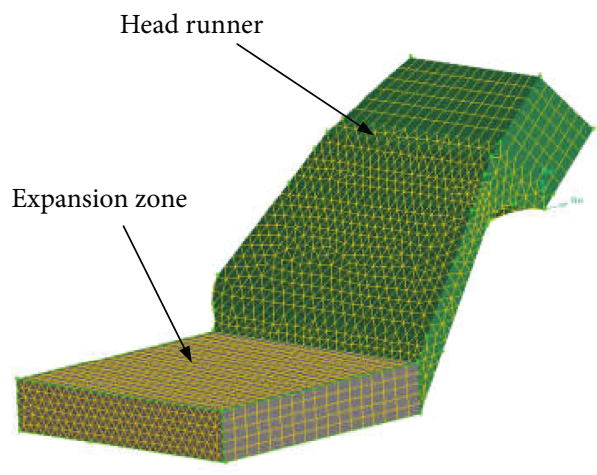

(a) The finite element model

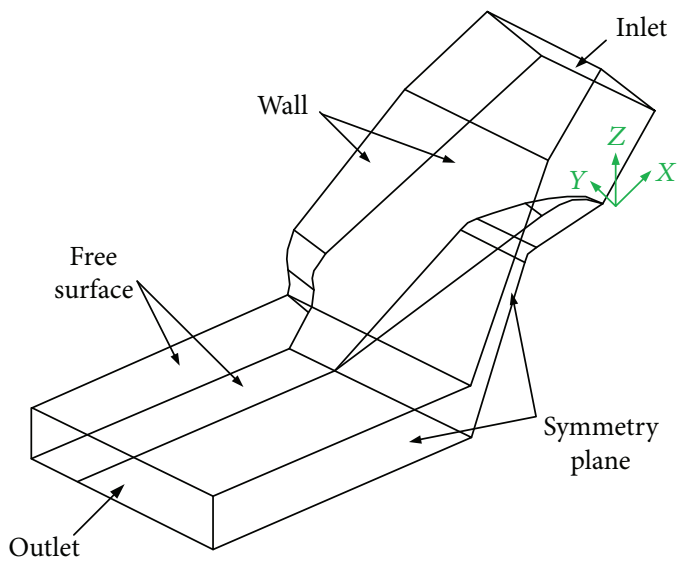

(b) Boundary conditions

FIgURE 2: Simulation model and boundaries.

The pure viscous Bird-Carreau model can effectively reflect the rheological properties of the rubber compound, and the Bird-Carreau model is applicable to low and high shear regions [14]. Therefore, the Bird-Carreau model is used as the constitutive equation. The relationship between the shear viscosity $\eta$ and the shear rate $\gamma$ is expressed as follows:

$$
\eta(\dot{\gamma})=\eta_{\infty}+\left(\eta_{0}-\eta_{\infty}\right)\left[1+(\lambda \dot{\gamma})^{2}\right]^{(n-1) / 2} .
$$

Here, $\eta_{0}$ is the zero-shear viscosity, $\eta_{\infty}$ the infinite shear viscosity, $\lambda$ the relaxation time, and $n$ the nonNewtonian index.

The rheological parameters of TQ387 rubber are determined using the RPA2000 rubber processing analyzer, and then the rheological parameters are fitted using the BirdCarreau model. Table 1 lists the specific constitutive model parameters at $110^{\circ} \mathrm{C}$.
TABLE 1: Bird-Carreau model parameters.

\begin{tabular}{lccc}
\hline$\eta_{0} /(\mathrm{Pa} \cdot s)$ & $\lambda / s$ & $n$ & $\eta_{\infty} /(\mathrm{Pa} \cdot s)$ \\
\hline 580064.5 & 24.19 & 0.162 & 0.002192 \\
\hline
\end{tabular}

\subsection{Boundary Conditions}

(1) Inflow boundary condition: assume full development of the rubber flow and inlet flow rate $Q=0.000345$ $\mathrm{m}^{3} / \mathrm{s}$.

(2) Symmetric boundary: tangential stress $f_{s}=0$ and normal velocity $v_{n}=0$.

(3) Wall boundary: low slip exists between the rubber compound and the wall in the actual extrusion process; thus, the Navier slip law is used to calculate the slip resistance as

$$
f(v)=-k\left(v-v_{\text {wall }}\right)^{e} .
$$


(4) Free boundary conditions: the free expansion surface must meet the conditions of the dynamics and kinematics boundary.

(5) Outlet boundary conditions: if no external traction is acting on the outlet, the normal force and tangential velocity of the surface are zero; that is, $f_{n}=0$ and $v_{s}=0$.

2.4. Numerical Algorithm. The velocity field is solved using the quadratic isoparametric element method, the pressure field by the linear isoparametric method, and the control equation by the Newton iteration method. The free zone is considered to have unknown deformation, and the rubber non-Newtonian index $n$ is small. Hence, the nonlinear problems in the simulation process are solved using the parameter evolution method, and the mesh of the free expansion zone is reset using the Optimesh-3D method.

\subsection{Results and Discussion}

2.5.1. Analysis of Die Swell of Rubber Compound. Figure 3 compares the ending cross-section profile between the physical extruder and simulation extruder. This figure shows that the rubber flow velocity was arranged after leaving the runner die. Because the flow velocity was high at the end and low in the middle, rubber die swell resulted in large deformation at the end and small deformation in the middle. Simultaneously, the end area was warped and the central area was smooth. The cross-sectional area obtained from physical extruder is $0.000465 \mathrm{~m}^{2}$; this was measured by using the TM-800 scanning laser cross-section measuring instrument. The cross-sectional area obtained from the simulation is $0.00451 \mathrm{~m}^{2}$; the simulation error was $3.1 \%$. This result indicates that the simulation results reflect the rubber flow phenomenon well and that the simulation method is reliable.

2.5.2. Analysis of Pressure Field in the Head Runner. Figure 4 illustrates the pressure simulation contour in the head runner. This figure shows that an obvious pressure gradient distribution occurs when the choke block obstructs the flow and forms a high-pressure range in the front region of the choke block. The high-pressure area results in increased energy consumption at the head. The minimum pressure areas appears at the outlet of runner area $D$ and choke block angle area $A$. Furthermore, negative pressure is clearly produced when rubber at the outlet of the runner and its flow is rearranged. The negative-pressure area extends along the profile of the choke block to the runner inside, thus affecting the stability of the rubber flow.

2.5.3. Analysis of Velocity Field in the Head Runner. Figure 5 shows the flow velocity simulation contour in the head runner. This figure shows that low velocity flow occurs when the choke block obstructs the flow. Furthermore, a stagnant flow region appears in the front region of the choke block angle $B$, and a no-flow region may be produced in the actual rubber extrusion process. If rubber is retained for a long time under high temperatures, vulcanization scorch may occur in the stagnating region, greatly affecting rubber extrusion quality.

The analyses shown in Figures 4 and 5 suggest that to improve the uniformity of the rubber compound flow state, the structural parameters of the head runner should be optimized to increase the extrusion quality while reducing energy consumption.

\section{Optimization Analysis and Discussion}

3.1. PB Experimental Design and Analysis. The shape and profile of the head runner greatly influence the rubber flow and extruder quality. To optimize the head runner structure and improve flow uniformity, the effect of each parameter of the runner on the flow uniformity should first be determined.

The PB experiment is based on the incomplete balance plate principle; it can obtain up to $(N-1)$ variables through $N$ experiments $[15,16]$. The main purpose of the two-level $\mathrm{PB}$ experimental design is to accurately estimate the major effects of the experimental factors through only a few tests.

In the extrusion process, the uniformity of the flow velocity distribution of rubber in the cross section of the runner outlet greatly influences the extrudate quality. To accurately describe the velocity uniformity of the extrudate in the cross section, the mean-square deviation of velocity (SDV) is used:

$$
\mathrm{SDV}=\sqrt{\frac{\sum_{i=1}^{n}\left(v_{i}-\bar{v}\right)^{2}}{n}} .
$$

Here, $v_{i}$ is the rubber flow velocity at node $I$ in the cross section of outlet to be researched, $\bar{v}$ is the average velocity for the selected nodes, and $n$ is the total number of chosen nodes. Therefore, the lower the SDV value, the better will be the extrudate quality. In this study, all outlet nodes are chosen for calculating the SDV. The number of nodes is 50 .

In the experimental analysis, the runner structure parameters are screened using the linear function and the interaction is neglected. The linear equation model is shown as

$$
Y=\beta_{0}+\sum \beta_{\mathrm{i}} x_{i} \quad(i=1,2,3, \ldots, 8) .
$$

Here, $Y$ is the SDV of the outlet, $x_{i}$ indicates the eight design parameters $A-H$, and $\beta_{i}$ is the regression coefficient indicating the effect of parameter $i$ on $Y$.

Tables 2 and 3 list the PB design factors and levels and the $\mathrm{PB}$ test results, respectively. By using the test results in Table 3, a regression analysis is performed to determine the effect of each parameter on SDV. Table 4 shows the results of the significance analysis of the effect of the head runner structure parameters on SDV.

According to the significance analysis result in the "Stdized effects" column of Table 4 , angles $A$ and $B$ and radii $F$ and $H$ have positive effects, whereas the remaining have negative effects. As per the analysis results shown in the "Contribution (\%)" column of Table 4, angles $A$ and $B$, outlet width $D$, and radius $C$ have a significant influence. However, the design of radius $C$ is directly affected by angles $A$ and $B$. Furthermore, outlet width $D$ is a key parameter affecting 


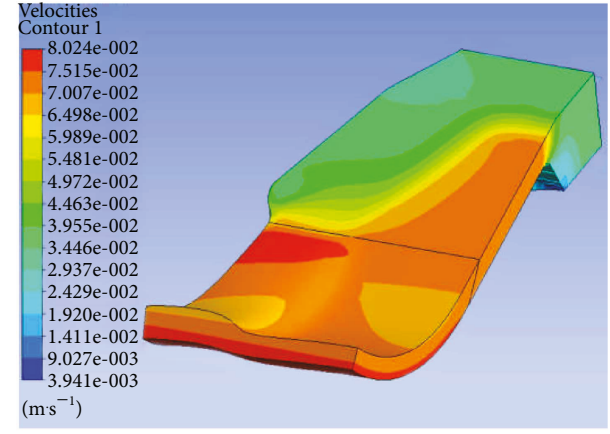

(a) Simulation result

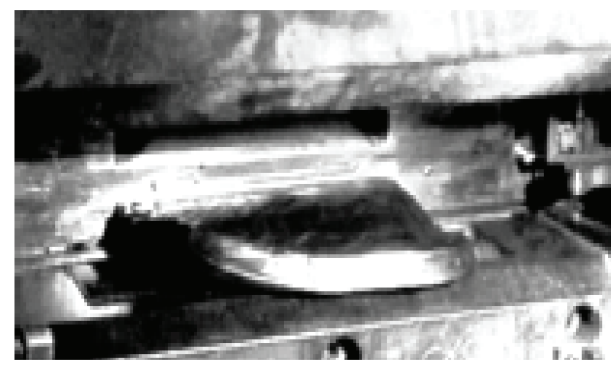

(b) Test result

FIgURE 3: Comparison of extrusion result between simulation and test.

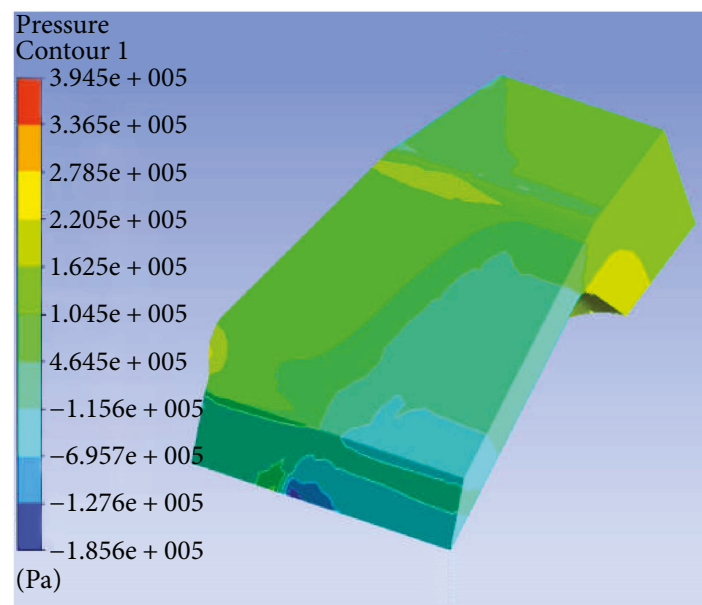

Figure 4: Pressure simulation contour in the head runner.

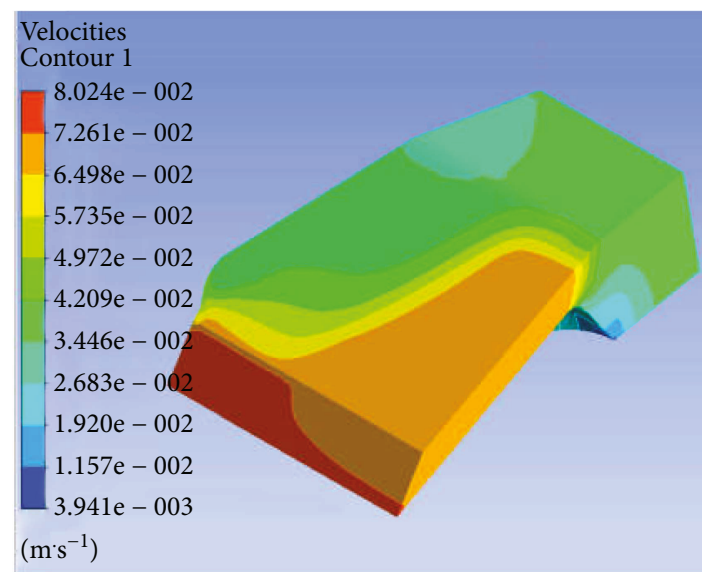

Figure 5: Velocity simulation contour in the head runner.

the rubber flow, and thus it is chosen as the key parameter affecting the SDV. The linear regression equation is given as

$$
Y=192.21+210.12 * A+79.59 * B-40.16 * D .
$$

3.2. Response Surface Methodology. The central composite design (CCD) is an experimental design method widely used to establish a second-order response surface model in process
TABLE 2: PB design factors and levels.

\begin{tabular}{lccc}
\hline Factors & Low level & High level & Original level \\
\hline Angle $A /^{\circ}$ & 10 & 20 & 15 \\
Angle $B /^{\circ}$ & 40 & 60 & 66 \\
Radius $C / \mathrm{mm}$ & 10 & 30 & 15 \\
Width $D / \mathrm{mm}$ & 55 & 85 & 70 \\
Radius $E / \mathrm{mm}$ & 15 & 35 & 25 \\
Radius $F / \mathrm{mm}$ & 20 & 40 & 30 \\
Width $G / \mathrm{mm}$ & 140 & 180 & 160 \\
Radius $H / \mathrm{mm}$ & 40 & 60 & 56.6
\end{tabular}

TABLe 3: PB test results.

\begin{tabular}{lccccccccc}
\hline \multirow{2}{*}{ Run } & & \multicolumn{1}{c}{ Factors } \\
& $A$ & $B$ & $C$ & $D$ & $E$ & $F$ & $G$ & $H$ & SDV \\
\hline 1 & 10 & 60 & 30 & 85 & 15 & 20 & 140 & 60 & 6.156 \\
2 & 10 & 40 & 10 & 55 & 15 & 20 & 140 & 40 & 6.358 \\
3 & 20 & 60 & 30 & 55 & 15 & 20 & 180 & 40 & 501.278 \\
4 & 10 & 40 & 30 & 55 & 35 & 40 & 140 & 60 & 7.131 \\
5 & 20 & 60 & 10 & 55 & 15 & 40 & 140 & 60 & 608.263 \\
6 & 20 & 40 & 30 & 85 & 15 & 40 & 180 & 60 & 306.007 \\
7 & 20 & 60 & 10 & 85 & 35 & 40 & 140 & 40 & 491.102 \\
8 & 10 & 60 & 30 & 55 & 35 & 40 & 180 & 40 & 8.676 \\
9 & 20 & 40 & 30 & 85 & 35 & 20 & 140 & 40 & 244.822 \\
10 & 10 & 40 & 10 & 85 & 15 & 40 & 180 & 40 & 8.053 \\
11 & 20 & 40 & 10 & 55 & 35 & 20 & 180 & 60 & 415.697 \\
12 & 10 & 60 & 10 & 85 & 35 & 20 & 180 & 60 & 9.300 \\
\hline
\end{tabular}

optimization studies [17]. CCD is an effective alternative to full-factorial design, which enables more data to be obtained through a smaller number of experiments. CCD differs from other methods by the use of axial points, defined as $\alpha$ values. Axial points require two more experiments for each parameter at the lowest and highest levels. All variables and their respective range were presented in Table 5. A five-levelthree-factor CCD leading to 20 experimental runs is used for optimizing the runner structure parameters. The experiment sequence was randomized to minimize the effects of uncontrolled factors. Table 6 shows the factorial design 
TABLE 4: Effect of factors on SDV.

\begin{tabular}{lcc}
\hline Factors & Stdized effects & Contribution $(\%)$ \\
\hline$A$ & 420.5 & 85.42 \\
$B$ & 106.12 & 5.45 \\
$C$ & -77.45 & 2.9 \\
$D$ & -80.33 & 3.12 \\
$E$ & -43.23 & 0.9 \\
$F$ & 40.94 & 0.81 \\
$G$ & -19.14 & 0.18 \\
$H$ & 15.38 & 0.11 \\
\hline
\end{tabular}

TABLE 5: Experimental variables and their coded levels for CCD.

\begin{tabular}{lccccc}
\hline \multirow{2}{*}{ Factors } & \multicolumn{5}{c}{ Coded variables levels } \\
& $-\alpha$ & -1 & 0 & 1 & $+\alpha$ \\
\hline$A$ & 6 & 10 & 14 & 18 & 22 \\
$B$ & 25 & 35 & 45 & 55 & 65 \\
$D$ & 40 & 52 & 64 & 76 & 88 \\
\hline
\end{tabular}

matrix and simulation results of each factorial experiment. The main effects and interactions among the factors were determined. The regression and variance were analyzed to determine the optimal process parameters.

The response surface methodology (RSM) consists of a set of mathematical and statistical techniques for developing a functional relationship between a response of interest $y$ and a number of associated control (or input or explanatory) variables $x_{1}, x_{2}, \ldots, x_{k}$. It is useful for applications in which reliable physical mathematical models to establish such a relationship are not available. It is also useful in cases where obtaining experimental data is costly to attempt to reduce the costs involved. In general, the relationship between $y$ and $x_{i}$ is unknown; however, it can be approximated by a low-degree polynomial model.

RSM focuses on using the experimental design to determine the variables that influence the response of interest [18]. After the experimental design, a response surface can be fitted so as to quantify the relationships between the response and the explanatory variables. This method is used as a guide to gradually vary the controllable factors that affect the response in such a way that the response value is improved.

In this study, we have used a second-order polynomial in keeping with Box and Wilson's study [19]. The model is represented in (7), where $x_{i}$ are the design variables, $\varepsilon$ is the residual, and $\beta_{0}$ to $\beta_{\mathrm{k}}$ are the regression parameters of the surface estimated from the experimental data.

$$
y=\beta_{0}+\sum_{i=1}^{k} \beta_{i} x_{i}+\sum_{i=1}^{k} \beta_{i i} x_{i}^{2}+\sum_{j=2}^{k} \sum_{i=1}^{j-1} \beta_{i j} x_{i} x_{j}+\varepsilon .
$$

Table 6 shows that the maximum and minimum values of SDV are 5.107 and 2.313, respectively, and its ratio is 2.235; this is smaller than the required ratio of 10 for RSM. From
TABLE 6: Composition of various runs of CCD and simulation results.

\begin{tabular}{|c|c|c|c|c|}
\hline \multirow{2}{*}{ Run } & \multicolumn{3}{|c|}{ Factors } & \multirow{2}{*}{ SDV } \\
\hline & $A$ & $B$ & $D$ & \\
\hline 1 & 18 & 55 & 52 & 5.107 \\
\hline 2 & 18 & 35 & 52 & 3.704 \\
\hline 3 & 10 & 55 & 52 & 2.724 \\
\hline 4 & 14 & 45 & 40 & 2.396 \\
\hline 5 & 14 & 45 & 64 & 2.435 \\
\hline 6 & 10 & 55 & 76 & 2.923 \\
\hline 7 & 6 & 45 & 64 & 3.081 \\
\hline 8 & 14 & 45 & 64 & 2.313 \\
\hline 9 & 18 & 35 & 76 & 3.832 \\
\hline 10 & 14 & 45 & 64 & 2.652 \\
\hline 11 & 14 & 65 & 64 & 2.846 \\
\hline 12 & 22 & 45 & 64 & 3.593 \\
\hline 13 & 14 & 45 & 64 & 2.347 \\
\hline 14 & 10 & 35 & 76 & 2.849 \\
\hline 15 & 14 & 45 & 64 & 2.634 \\
\hline 16 & 18 & 55 & 76 & 5.257 \\
\hline 17 & 10 & 35 & 52 & 2.638 \\
\hline 18 & 14 & 45 & 64 & 2.313 \\
\hline 19 & 14 & 25 & 64 & 2.558 \\
\hline 20 & 14 & 45 & 88 & 2.918 \\
\hline
\end{tabular}

the data in Table 6, the response surface function between SDV and the three key factors is obtained as follows:

$$
\begin{aligned}
R= & 14.722-0.776 * A-0.22 * B-0.105 * D+0.0083 \\
& * A * B+0.018 * A^{2}+0.001398 * B^{2}+0.001 * D^{2} .
\end{aligned}
$$

Table 7 shows the quadratic model variance analysis of fitting the experimental results in response surface analysis. The $F$ value is 4.97 , and the multiple correlation coefficient is $R^{2}=0.816$, indicating good fit between the calculated variance of the velocity variance and the predicted value at the extrusion of the compound.

Figure 6 illustrates the effects of angles $A$ and $B$ on SDV. In general, the SDV increases with an increase in angle $B$ when angle $A$ was less than $10^{\circ}$, whereas it decreases with an increase in angle $B$ when angle $A$ was more than $10^{\circ}$. At a high angle $B$, the increase in angle $A$ has a significant effect on SDV. However, at high angle $B$, the increase in angle $A$ resulted in an increase in SDV. At a low angle $B$, SDV first decreases and then increases. SDV is higher for high angle $A$ and high angle $B$. Figure 6 also shows a clear interaction between angles $A$ and $B$. The combination of different values of angles $A$ and $B$ significantly affects the SDV. Figure $7 \mathrm{dem}$ onstrates the effects of angle $A$ and outlet width $D$ on the SDV; this figure shows that angle $A$ should be set at a low value for improving the uniformity of the rubber flow in the outlet cross section. A high range of angle $A$ resulted in high SDV at all values of angle $B$; with an increase in angle 
TABLE 7: Variance analysis of objective functions.

\begin{tabular}{lccccc}
\hline Source & $\begin{array}{c}\text { Free } \\
\text { degree }\end{array}$ & $\begin{array}{c}\text { Sum of } \\
\text { squares }\end{array}$ & $\begin{array}{c}\text { Mean } \\
\text { square }\end{array}$ & $\begin{array}{c}F \\
\text { value }\end{array}$ & $\begin{array}{c}P \\
\text { value }\end{array}$ \\
\hline Fit & 9 & 0.170 & 0.019 & 4.97 & 0.012 \\
Residual & 10 & 0.041 & 0.004 & & \\
$\begin{array}{l}\text { Lack of } \\
\text { fit }\end{array}$ & 5 & 0.037 & 0.007 & 11.2 & 0.0093 \\
Sum & 19 & 0.208 & & & \\
\hline
\end{tabular}

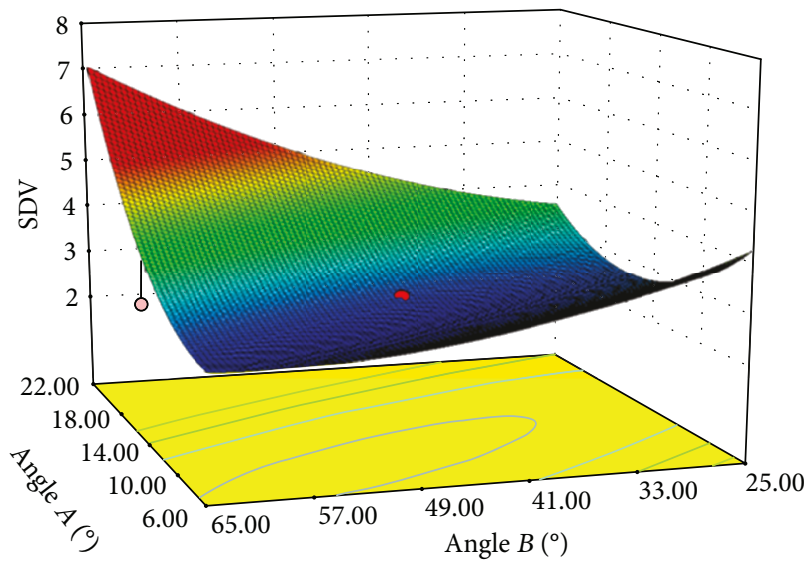

FIGURE 6: Response surface plots of the SDV as a function of angles $A$ and $B$.

$A$, SDV first decreased and then increased. Nevertheless, the effect of outlet width $D$ on the SDV at high angle $A$ is greater than that at low angle $A$. The lowest SDV was observed at low angle $A$ of $10^{\circ}$ and at outlet width $D$ of $56 \mathrm{~mm}$. Figure 8 shows the effects of angle $B$ and outlet width $D$ on the SDV. This figure shows that the SDV increases, regardless of whether angle $B$ and outlet width $D$ increase or decrease simultaneously. The gradient change in the level surface indicates that the interaction between angle $B$ and outlet width $D$ does not have a significant effect on the SDV. If angle $A$ remains low, angle $B$ and outlet width $D$ should be kept low to improve the uniformity of the rubber flow.

3.3. Simulated Annealing (SA) Algorithm. If the minimal function is obtained using (8), the problem becomes one of nonlinear optimization. Metropolis, Rosenbluth, and Teller originally proposed the simulated annealing (SA) algorithm to simulate the annealing process [20]. SA starts with a high temperature. After generating an initial solution, it attempts to move from the current solution to one of its neighboring solutions. SA algorithm uses the analogy between the minimization of the cost function of an optimization problem and the slow procedure of gradually cooling a metal until it reaches its "freezing" point, at which the energy of the system has acquired a globally minimal value. This algorithm is based on an iterative method proposed by Kiani and Yildiz that simulates the transition of atoms in equilibrium at a given temperature [21].

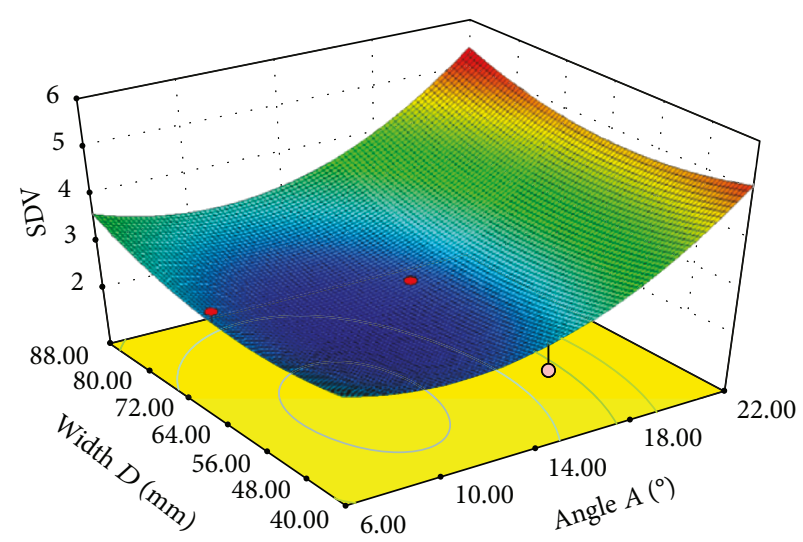

FIGURE 7: Response surface plots of the SDV as a function of outlet width $D$ and angle $A$.

For the combinatorial optimization problem, each point in the solution space represents a target function solution. The so-called optimization involves finding the extremum of the target function in the solution space. If the objective function is the energy function, the control parameter of the combinatorial problem is the temperature and solution space is the state space. The aim then is to find the base state by using the SA algorithm; this can be seen as target function extremum optimization. Consider the rubber flow velocity variance at the outlet as the target and set the constraint conditions as angle $A=6^{\circ}-25^{\circ}$, angle $B=20^{\circ}-70^{\circ}$, and outlet width $D=30 \mathrm{~mm}-90 \mathrm{~mm}$. The temperature renewal function in simulation annealing is then given by the following:

$$
t_{k}=\frac{t_{0}}{\ln (1+k)} .
$$

Here, $k$ is the temperature cycle number, $t_{k}$ is the temperature at step $k$, and $t_{0}$ is the initial temperature. The state receptive function is given by the following:

$$
p_{i j}=\left\{\begin{array}{cc}
1, & \Delta E \geq 0, \\
\frac{1}{1+\exp \left(\Delta E / t_{k}\right)}, & \Delta E<0 .
\end{array}\right.
$$

Here, $\Delta E=E_{i}-E_{j}, E_{i}$ is the energy function in the current solution state and $E_{j}$ is the energy function in receptive function state. The use of $P_{i j}$ forces the systems to evolve into thermal equilibrium; that is, after a large number of perturbations, the probability distribution of the states approaches the Boltzmann distribution. The energy function is the target function given by (8).

The searching mode for the receptive solution space is based on the small pace search method as expressed in the following:

$$
X_{j}=X_{i}+l \Delta .
$$

Here, $X_{i}$ is the current state solution, $X_{j}$ is the new state solution, $l$ is the searching cycle number, and $\Delta$ is the searching step. 
The proposed SA algorithm can be described as follows:

Step 1. Design the algorithm parameters. Determine the initial value of temperature $t_{0}$ and set $t=t_{0}$. Randomly find an initial feasible solution $X_{0}$ that is assigned as the current solution $X_{i}$. Set iteration counter $k=1$.

Step 2. Calculate the energy function at the current solution and obtain the new state solution $X_{j}$ based on (11).

Step 3. Calculate the new energy function $E\left(X_{j}\right)$ and obtain the energy difference $\Delta E$.

Step 4. The judgment standard of the new solution is accepted or is not according to (10). If it is accepted as the new trial solution, set $X_{i}=X_{j}$ and go to step 6; otherwise, go to step 5 .

Step 5. If the frequency search reaches the upper limit, go to step 8; otherwise, $l=l+1$ and go to step 2 .

Step 6. $k=k+1$; the new temperature $t_{k}$ is obtained using (9).

Step 7. If the temperature $t_{k}$ reaches its minimum, go to step 8; otherwise, go to step 2.

Step 8. End.

The search for the minimal energy function is executed by setting the initial temperature as $3000^{\circ} \mathrm{C}$, initial minimum temperature as $0.05^{\circ} \mathrm{C}$, searching step as 0.01 , and maximum number of loops in interval searching as 1000 . The optimal solution of the energy function is $A=10.8^{\circ}, B=46.6^{\circ}$, and $D=52.5 \mathrm{~mm}$. Then, the minimum $R$ value is 2.15 .

3.4. Verification of Numerical Experiment. To further verify the improvement in extrusion quality, an optimized head runner was built with angle $A=10.8^{\circ}$, angle $B=46.6^{\circ}$, and outlet width $D=52.5 \mathrm{~mm}$. The other structure parameters of the original head runner were maintained.

By considering the velocity variance of the rubber flow in the outlet cross section as the target, the rheological parameters of rubber were tested at $120^{\circ} \mathrm{C}, 100^{\circ} \mathrm{C}$, and $90^{\circ} \mathrm{C}$. The effect of temperature on the extrudate quality of optimal runner was analyzed under inlet flow rate of $0.000345 \mathrm{~m}^{3}$. By changing the inlet flow rate to $0.000345 \mathrm{~m}^{3}, 0.0004 \mathrm{~m}^{3}$, and $0.00029 \mathrm{~m}^{3}$ at $110^{\circ} \mathrm{C}$, the effect of the inlet flow rate on the extrudate quality of optimal runner was analyzed at $110^{\circ} \mathrm{C}$. Table 8 lists the obtained results, which indicate that the optimized runner can reduce the flow velocity variance and improve the rubber extrudate quality under different temperatures and different inlet flow rates.

Figure 9 shows the flow velocity distribution in the optimized runner at $110^{\circ} \mathrm{C}$ and at $0.000345 \mathrm{~m}^{3}$ inlet flow rate. The flow velocity distribution was uniform in the outlet cross section, and the velocity gradient in the flow direction was reduced significantly. Figure 10 shows the extrusion state of rubber in the optimal runner. This figure shows that the

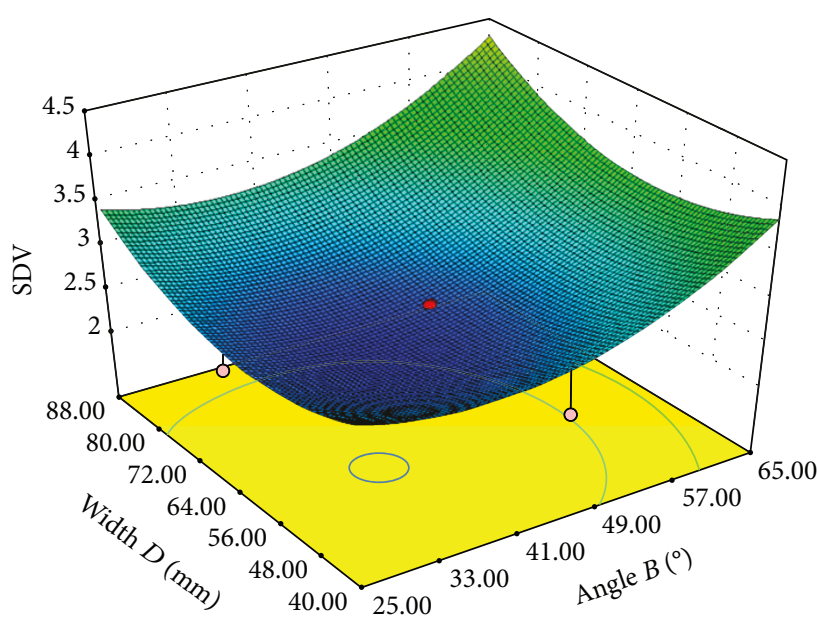

FIgURE 8: Response surface plots of the SDV as a function of outlet width $D$ and angle $B$.

TABLE 8: SDV at different temperatures and flow ratios.

\begin{tabular}{lccccccc}
\hline \multirow{2}{*}{ Conditions } & \multicolumn{3}{c}{ Temperature/ ${ }^{\circ} \mathrm{C}$} & \multicolumn{4}{c}{ Flow rates $\times 10^{-4}$ ' } \\
& 120 & 110 & 100 & 90 & 2.9 & 3.45 & 4 \\
\hline Original & 3.14 & 3.12 & 3.31 & 3.93 & 2.71 & 3.12 & 3.54 \\
Optimization & 2.19 & 2.69 & 3.27 & 3.73 & 2.28 & 2.69 & 2.71 \\
\hline
\end{tabular}

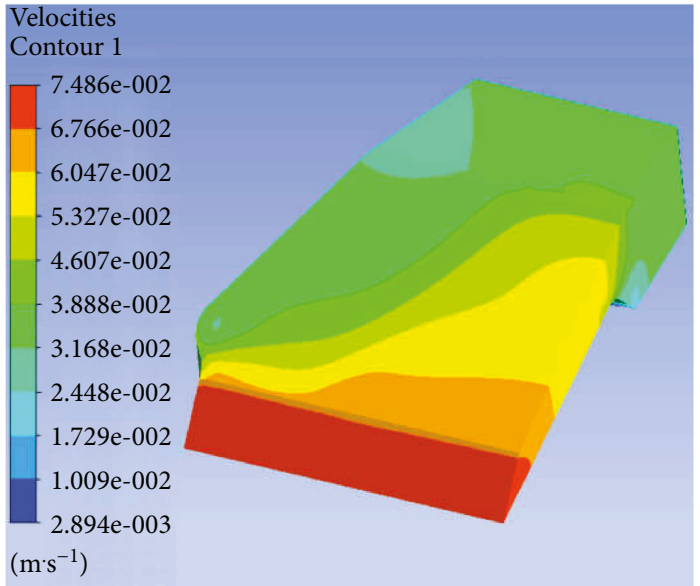

FIGURE 9: Rubber flow velocity in optimized runner at $110^{\circ} \mathrm{C}$ and at $0.000345 \mathrm{~m}^{3}$ inlet flow rate.

rubber flow is stable and that warping disappeared. The extrudate quality of rubber was improved.

\section{Summary}

(1) By simulating the extrusion of rubber, the choke block and runner were found to significantly influence the exit velocity of rubber. Therefore, $\mathrm{PB}$ experimental results indicate that angles $A$ and $B$ and outlet width $D$ of the runner are the key factors affecting the extrudate quality considerably. The radius $C$, 


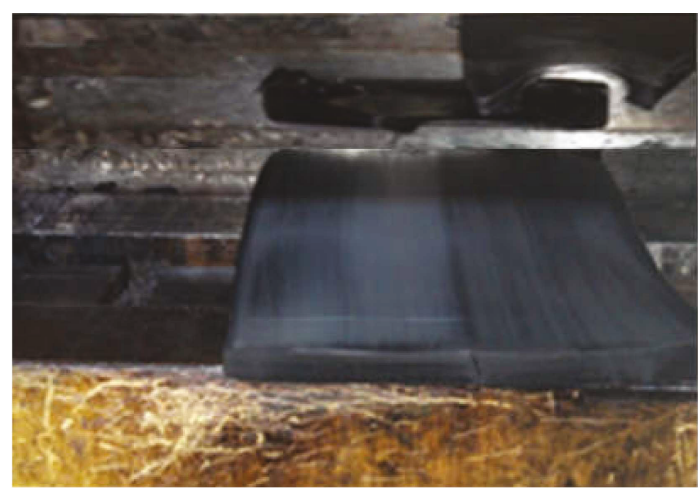

Figure 10: Extrusion state of rubber using optimal runner at $110^{\circ} \mathrm{C}$ and at $0.000345 \mathrm{~m}^{3}$ inlet flow rate.

outlet width $D$, radius $E$, and inlet width $G$ have negative effects, whereas the remaining factors have positive effects.

(2) The CCD experimental method was used to construct the response surface model of the three key parameters, namely, angles $A$ and $B$ and outlet width $D$, and to obtain the influence law of combinations of different parameters on the uniformity of the rubber flow. The optimal key factors of the runner are obtained through SA optimization. The simulation results show that relatively more uniform velocity distribution of rubber flow in the outlet cross section is achieved.

(3) Through a comparative analysis of the compound extrusion quality modeling at different temperatures and the inlet flow rate in the optimized runner, the flow velocity distribution is improved. The test results of the rubber extrusion state by using an optimized runner indicate that the optimized runner significantly improves the extrusion quality. Thus, the proposed optimization method is effective for extrusion runner design.

(4) The proposed optimization method is suitable for optimizing the extrusion runner as well as for other extrusion experiments. The optimization strategy for the head runner can provide theoretical guidelines for improving tire rubber extrusion and coextrusion processing technology.

\section{Data Availability}

The data used to support the findings of this study are available from the corresponding author upon request.

\section{Conflicts of Interest}

The authors declare that there is no conflict of interest regarding the publication of this paper.

\section{Acknowledgments}

The authors are grateful for the financial support by the National Natural Science Foundation of China (Grant no. 51605198 and 51675240), Jiangsu Provincial Funds for Young Scientists (Project no. BK20160528); Jiangsu Provincial Six Talent Peaks (Project no. JXQC-011), and the Jiangsu University Advanced Talents Initial Funding Project (1291120046).

\section{References}

[1] W. Michaeli, Extrusion Dies for Plastics and Rubber: Design and Engineering Computations, Carl Hanser Verlag GmbH \& Co. KG, Germany, 2003.

[2] Z. Jiang, T. Ouyang, X. Yao, and Y. Fei, "Die swell behavior of liquid crystalline mesophase pitch," Journal of Materials Science, vol. 51, no. 15, pp. 7361-7369, 2016.

[3] A. N. Gent and J. D. Walter, The Pneumatic Tire, U.S. Department of Transportation National Highway Traffic Safety Administration, Washington, 2006.

[4] S. G. Kim, S. P. Yoon, S. W. Nam, S. H. Hyun, and S. A. Hong, "Fabrication and characterization of a YSZ/YDC composite electrolyte by a sol-gel coating method," Journal of Power Sources, vol. 110, no. 1, pp. 222-228, 2002.

[5] X. Sun, G. Zhao, C. Zhang, Y. Guan, and A. Gao, "Optimal design of second-step welding chamber for a condenser tube extrusion die based on the response surface method and the genetic algorithm," Materials and Manufacturing Processes, vol. 28, no. 7, pp. 823-834, 2013.

[6] J. Dooley and B. T. Hilton, "Layer rearrangement in coextrusion," Plastics Engineering, vol. 50, no. 2, pp. 25-27, 1994.

[7] J. Musil and M. Zatloukal, "Effect of die exit geometry on internal die drool phenomenon during linear HDPE melt extrusion," International Journal of Heat and Mass Transfer, vol. 56, no. 1-2, pp. 667-673, 2013.

[8] S. Elgeti, M. Probst, C. Windeck, M. Behr, W. Michaeli, and C. Hopmann, "Numerical shape optimization as an approach to extrusion die design," Finite Elements in Analysis and Design, vol. 61, pp. 35-43, 2012.

[9] Y. Zhong, H. S. Liu, Y. B. Huang, K. Zhang, and T. Li, “Optimizaiton analysis of extruder flow channel on UHMWPE during extrusion process," Plastics, vol. 44, no. 6, pp. 85-88, 2015.

[10] L. I. N. Li-hong, J. I. A. Xiao-yan, and M. A. Tir-jun, "Design of extruder flow channel based on fluent," China Mechanical Engineering, vol. 27, no. 7, pp. 944-950, 2016, (in Chinese).

[11] D. A. I. Yuan-kan, L. I. Xiang-yang, and Z. H. O. U. Chi-xing, "Optimizing design of extrusion flow channel of rubber sealing strip by computer simulation," China Synthetic Rubber Industry, vol. 31, no. 2, pp. 92-95, 2008, (in Chinese).

[12] C. Liang, L. Wei, B. Jiang, G. R. Li, and G. L. Wang, "Design of tire compounds coextrusion die based on numerical simulation," Journal of Mechanical Engineering, vol. 49, no. 22, pp. 103-108, 2013.

[13] E. Mitsoulis, M. Battisti, A. Neunhäuserer et al., "Flow behaviour of rubber in capillary and injection moulding dies," Plastics, Rubber and Composites, vol. 46, no. 3, pp. 110-118, 2017.

[14] J. H. Kim and M. Y. Lyu, "Predictions of flow behaviors and entrance pressure drop characteristics of a rubber compound in a capillary die using various rheological models," Polymer Engineering \& Science, vol. 54, no. 10, pp. 2441-2448, 2014. 
[15] S. Dayana Priyadharshini and A. K. Bakthavatsalam, "Optimization of phenol degradation by the microalga Chlorella pyrenoidosa using Plackett-Burman design and response surface methodology," Bioresource Technology, vol. 207, pp. 150-156, 2016.

[16] L. Ma, L. Wang, J. Tang, and Z. Yang, "Optimization of arsenic extraction in rice samples by Plackett-Burman design and response surface methodology," Food Chemistry, vol. 204, pp. 283-288, 2016.

[17] M. Alizadeh and S. M. Sadrameli, "Numerical modeling and optimization of thermal comfort in building: central composite design and CFD simulation," Energy and Buildings, vol. 164, pp. 187-202, 2018.

[18] C. Ai, A. Wu, Y. Wang, and C. L. Hou, "Optimization and mechanism of surfactant accelerating leaching test," Journal of Central South University, vol. 23, no. 5, pp. 1032-1039, 2016.

[19] H. Gao, G. Bai, Y. Gao, and T. W. Bao, "Reliability analysis for aeroengine turbine disc fatigue life with multiple random variables based on distributed collaborative response surface method," Journal of Central South University, vol. 22, no. 12, pp. 4693-4701, 2015.

[20] A. Corana, M. Marchesi, C. Martini, and S. Ridella, "Minimizing multimodal functions of continuous variables with the "simulated annealing" algorithm," ACM Transactions on Mathematical Software, vol. 13, no. 3, pp. 262-280, 1987.

[21] M. Kiani and A. R. Yildiz, "A comparative study of nontraditional methods for vehicle crashworthiness and $\mathrm{NVH}$ optimization," Archives of Computational Methods in Engineering, vol. 23, no. 4, pp. 723-734, 2016. 


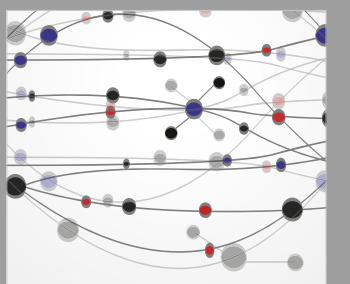

The Scientific World Journal
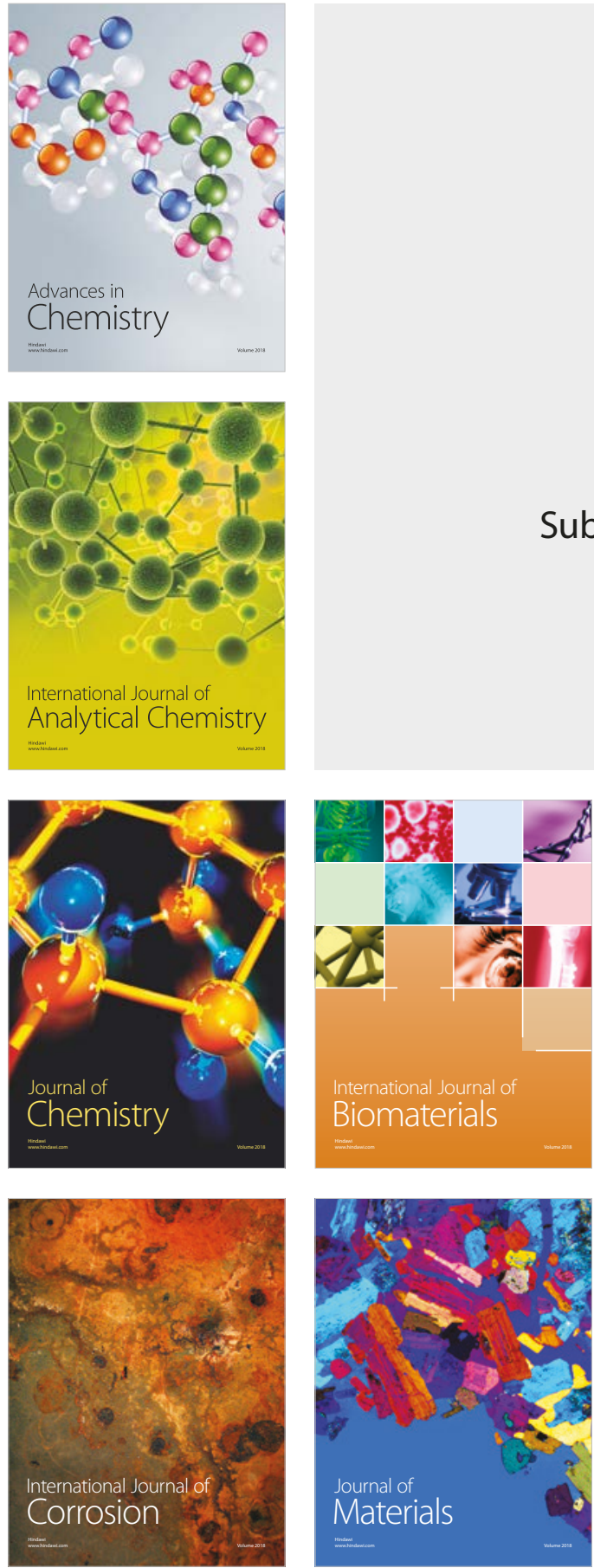

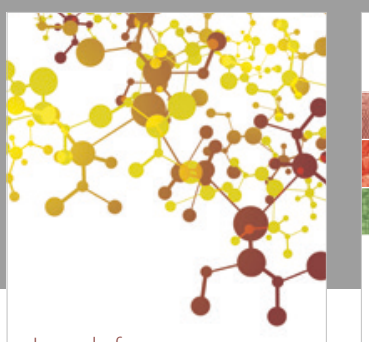

Journal of

Applied Chemistry
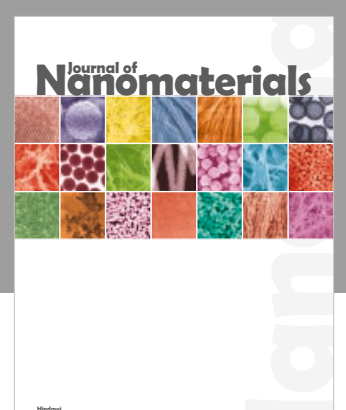

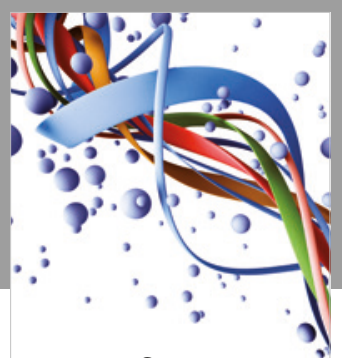

Scientifica

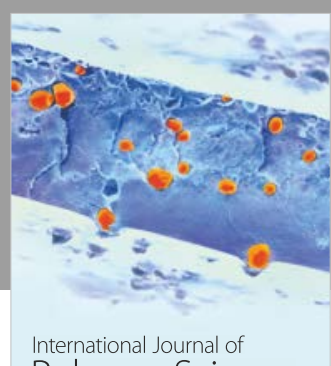

Polymer Science

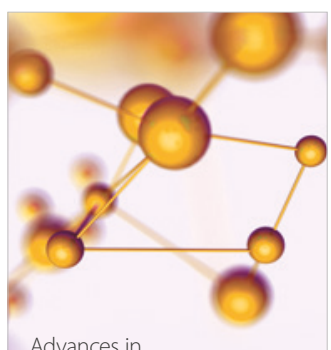

Physical Chemistry
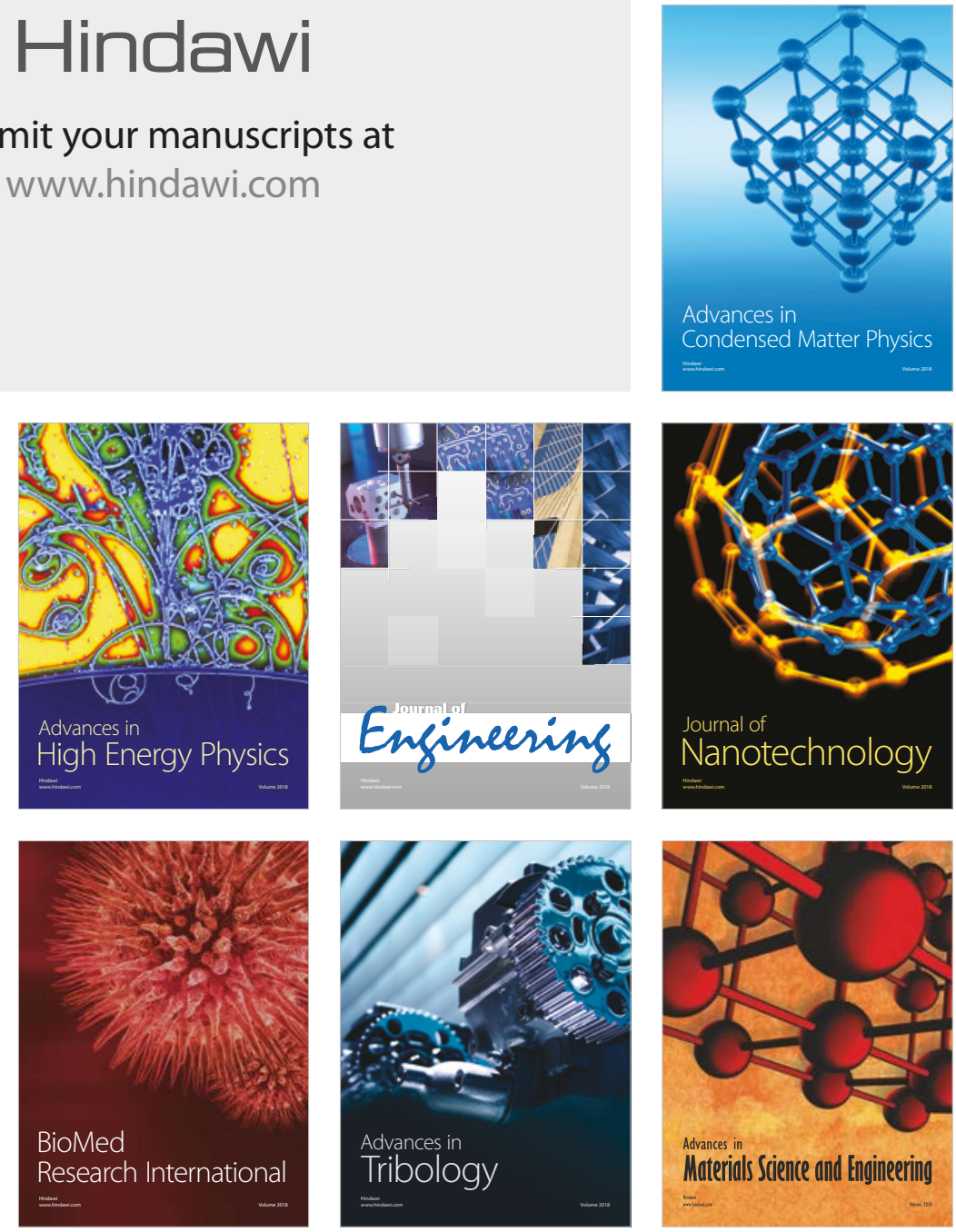